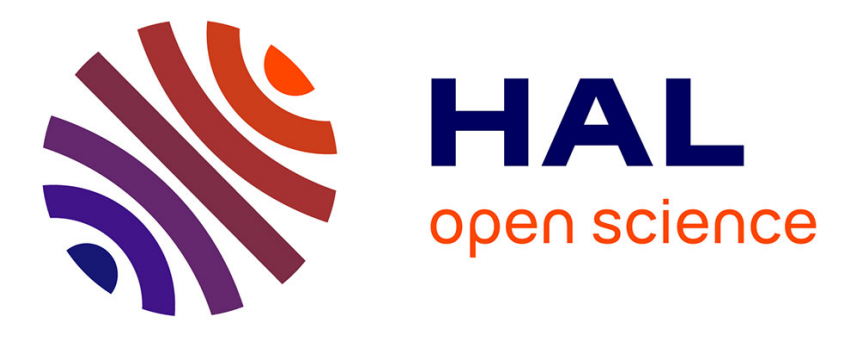

\title{
AOPDF-shaped optical parametric amplifier output in the visible
}

Antoine Monmayrant, Arnaud Arbouet, Bertrand Girard, Béatrice Chatel, A. Barman, B. J. Whitaker, D. Kaplan

\section{- To cite this version:}

Antoine Monmayrant, Arnaud Arbouet, Bertrand Girard, Béatrice Chatel, A. Barman, et al.. AOPDF-shaped optical parametric amplifier output in the visible. Applied Physics B - Laser and Optics, 2005, 81, pp.177-180. 10.1007/s00340-005-1890-6 . hal-00004945

\section{HAL Id: hal-00004945 https://hal.science/hal-00004945}

Submitted on 21 May 2005

HAL is a multi-disciplinary open access archive for the deposit and dissemination of scientific research documents, whether they are published or not. The documents may come from teaching and research institutions in France or abroad, or from public or private research centers.
L'archive ouverte pluridisciplinaire HAL, est destinée au dépôt et à la diffusion de documents scientifiques de niveau recherche, publiés ou non, émanant des établissements d'enseignement et de recherche français ou étrangers, des laboratoires publics ou privés. 


\title{
AOPDF-shaped optical parametric amplifier output in the visible
}

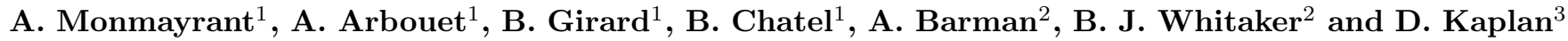 \\ 1 Laboratoire Collisions, Agrégats Réactivité (CNRS UMR 5589), IRSAMC Université Paul Sabatier, 31062 Toulouse, France \\ 2 School of Chemistry, University of Leeds, Leeds, LS2 9JT, UK \\ 3 Fastlite, Batiment 403, Ecole Polytechnique, Palaiseau, France
}

The date of receipt and acceptance will be inserted by the editor

\begin{abstract}
Time Shaping of ultrashort visible pulses has been performed using a specially designed AcoustoOptic Programmable Dispersive Filter of $50 \%$ efficiency at the output of a two-stage noncollinear optical parametric amplifier. The set-up is compact and reliable. It provides a tunable shaped source in the visible with unique features: 4 ps shaping window with preserved tunability over $500-650 \mathrm{~nm}$, and pulses as short as 30 fs. Several $\mu \mathrm{J}$ output energy is easily obtained.
\end{abstract}

The development of femtosecond laser technology has I opened access to unforeseen applications in molecular, chemical physics, as well as biology 1,2,3,4. In the past ten years, the active control of ultrafast physical or chemical processes by means of well-defined shaped laser pulses has become possible [5,6]. The high number of potential applications of femtosecond pulse shaping turned it very quickly into a very intense field of research.

Traditional methods for femtosecond pulse shaping are based on a Liquid Crystal Device (LCD) or Acousto$\checkmark$ Optic Modulator (AOM) placed in the Fourier plane of a grating based zero dispersion $4 \mathrm{f}$ configuration $[7,8,9]$. The different wavelengths are spatially separated and can then be addressed individually. Spectacular results have been obtained with such devices [10 11]. However, the pixelation causes pre- and post-pulses in the time domain containing sometimes a substantial fraction of the total pulse energy 12. Changing the wavelength requires careful realignment, thus precluding easy tunability. Finally, their large size can be a severe limitation in some applications.

Visible shaped pulses have been obtained by inserting such pulse shapers in a Noncollinear Optical Parametric Amplifier (NOPA) 13,14. Either the white light seed was shaped with a LCD [13, or the output of the first stage of a two-stage NOPA was shaped with an AOM 14. Despite significant results, the complexity of the set-up was increased dramatically and little tunability was available. Also the constraint of temporal overlap between shaped and pump pulses reduced the shaping window to ca 200 fs 13 or 1 ps 14. In other studies, the $4 \mathrm{f}$ shaper was placed directly at the output of a visible laser 4, 15, 16, 17. A much simpler scheme, avoiding the complexity of the $4 \mathrm{f}$ line, and with higher efficiency, can be achieved using instead an Acousto-Optic Programmable Dispersive Filter (AOPDF) [18] at the output of a NOPA.

AOPDF are based on the propagation of light in an acousto-optic birefringent crystal. The interaction of an incident ordinary optical wave with a collinear acoustic shear wave leads to diffraction of an extraordinary wave. Spectral phase and amplitude pulse shaping of a femtosecond optical pulse can be achieved by controlling the amount of extraordinary versus ordinary propagation in the optical path of each of its spectral components. The collinear acousto-optic interaction and the reduced size result in an easy-to-align device, appropriate for insertion in an amplified laser chain or in a pump-probe setup. AOPDF have proven to be very useful to correct the time aberrations introduced in Chirped Pulse Amplifiers, for amplitude and phase control of ultrashort pulses [18 19], or even in characterization set-ups [20].

Most of AOPDF applications have been restricted so far to the near infrared. Indeed, phase-matching conditions for shorter wavelengths require higher acoustic frequencies for which absorption is increased. Actually, preliminary experiments demonstrated that acoustic absorption is predominant in the blue region of the spectrum 21. To overcome these limitations, we have specially designed a new AOPDF accepting pulse energy densities up to $300 \mu \mathrm{J} / \mathrm{cm}^{2}$ with reduced absorption at wavelengths as low as $500 \mathrm{~nm}$.

Here, we report on pulse compression and shaping directly at the output of a home made NOPA with this new AOPDF. This results in a simple, compact, and reliable device providing sub-30fs pulses on a 4 ps shaping window, easily tunable in the 500-650 $\mathrm{nm}$ range.

The design of the AOPDF must fulfill two requirements: the optical yield has to be maximum because any 
loss will not be recovered in further amplification stages and the shaping capabilities of the device must be preserved. In particular, the device should compensate for its own basic dispersion (due to the wavelength dependent refractive index). Otherwise, obtaining the shortest pulses will require an additional compressor device, undermining the simplicity of the approach.

The acoustic beam orientation being set to align the group velocity of both acoustic and optical beams, the main design parameter is the angle of propagation of the latter, $\theta=([110], k)$, in the birefringent crystal $\mathrm{Te}_{2}$.

The diffraction efficiency (i.e. the fraction of the energy at a given optical wavelength recovered in the diffracted beam) has a maximum at $\theta=58.5^{\circ}$ and decreases at smaller angles 22 .

The maximum programmable delay for an incoming pulse centered at $\lambda_{o p t}$ is:

$$
T_{\max }=\Delta n_{g}\left(\lambda_{o p t}\right) \cos ^{2}(\theta) L / c
$$

where

$$
\Delta n_{g}\left(\lambda_{o p t}\right)=n_{g, e}\left(\lambda_{o p t}\right)-n_{g, o}\left(\lambda_{o p t}\right)
$$

is the group birefringence, $\mathrm{L}$ the length of the crystal and c the speed of light. Part of this delay capacity, called $T_{\text {comp }}$, will be used to compensate the dispersion of the device and the remaining delay $T_{\max }-T_{\text {comp }}$ available for pulse shaping is a decreasing function of $\theta$.

The acoustic absorption $\beta$ in the crystal has a quadratic dependence on the acoustic frequency $f$ and a non-trivial dependence on $\theta$ [23]:

$$
\beta=\mathcal{C} \frac{\gamma^{2}(\theta) f^{2}(\theta)}{V^{4}(\theta)}
$$

$\mathcal{C}$ is a constant, $V$ is the acoustic phase velocity, and $\gamma$ the Gruneisen constant. A $\beta$ value of $18 \mathrm{~dB} / \mu \mathrm{s} . \mathrm{GHz}^{2}$ has been measured experimentally 22 for $\theta=0^{\circ}$. Although a precise determination of the $\gamma(\theta)$ law is difficult, one can infer that $\beta$ decreases with $\theta$.
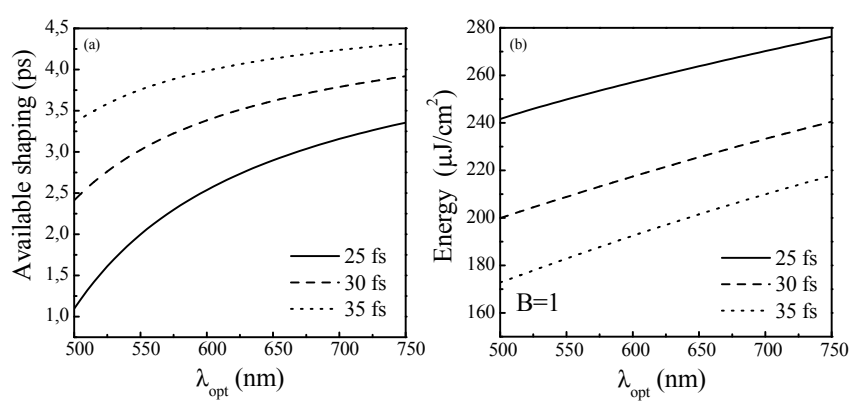

Fig. 1 Pulse shaping capability (a) and intensity capability (b) of the AOPDF for different FWHM Fourier limited pulse durations, as a function of wavelength.

Taking into account these constraints, a $25 \mathrm{~mm}$ crystal at $\theta=45^{\circ}$ has been designed giving excellent results in terms of shaping capacities and optical output power as described below. The computed temporal width of the shaping window is depicted in Fig. 1a as a function of the optical wavelength for several FWHM pulse durations. The AOPDF allows shaping on up to 4 ps. The propagation of 30 fs pulses through the whole AOPDF yields B integrals (accumulated self phase modulation) of unity for intensities above $200 \mu \mathrm{J} / \mathrm{cm}^{2}$ on the $500-750$ nm range (Fig. 1b).

Experiments have been performed using this newly designed AOPDF at the output of a two-stage NOPA 24 25]. The output beam of the NOPA was split into two parts as shown in Fig. 2. One part was compressed in a silica prism compressor, leading to durations around $25-30$ fs on the $500-650 \mathrm{~nm}$ range. This value is close to $20 \mathrm{fs}$ which is the Fourier Transform limited pulse duration corresponding to the FWHM of the intensity spectrum. The other part remained uncompressed and was fed directly in the AOPDF. The beam profile is adjusted so that its FWHM lies from 1.5 to $2.5 \mathrm{~mm}$. Using a variable neutral density filter, the energy in front of the AOPDF was varied from 1 to $6 \mu \mathrm{J}$, corresponding to energy densities up to $300 \mu \mathrm{J} / \mathrm{cm}^{2}$ without any major self phase modulation effect, a value somewhat higher than the theoretical one. Pulses coming out of the AOPDF are characterized either by 2 nd order autocorrelation or by cross-correlation with the compressed reference beam (see Fig. 2).

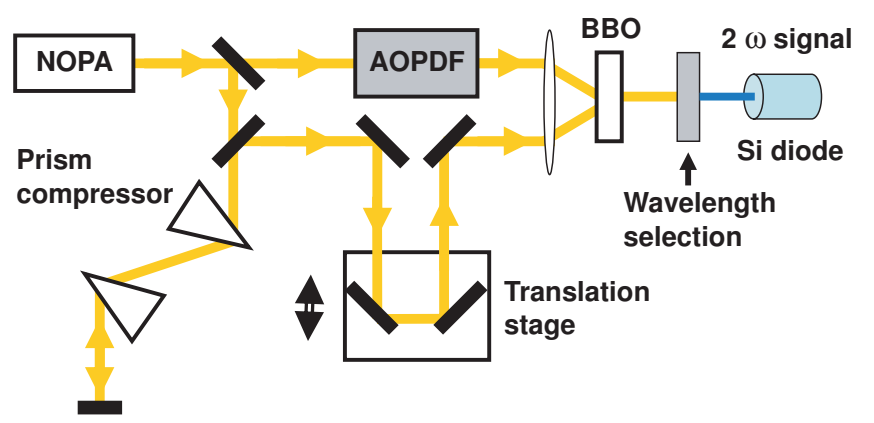

Fig. 2 Experimental setup. NOPA output $12 \mu \mathrm{J}$ (pumped with $800 \mathrm{~nm}, 120 \mathrm{fs}, 250 \mu \mathrm{J}$ pulses).

In a first series of experiments, acoustic waves allowing compensation of the quadratic phase term including the pulse initial chirp $\left(\sim 1000 \mathrm{fs}^{2}\right)$ and $\mathrm{Te}_{2}$ induced chirp $\left(\sim 25000 \mathrm{fs}^{2}\right)$ together with higher order phase terms have been programmed. This lead to compression of the output of the NOPA down to sub-30 fs pulses on the 500-650 nm range.

Fig. 3 shows the spectrum after the AOPDF at 510, 550 and $640 \mathrm{~nm}$ together with second harmonic intensity autocorrelation in a $100 \mu \mathrm{m}$ BBO. The FWHM is sub30 fs assuming a sech ${ }^{2}$ pulse profile. Additional experiments performed using a similar set-up where the NOPA is a commercial Clark-MXR NOPA confirmed that the small pedestal at $510 \mathrm{~nm}$ in Fig. 3 could be reduced. The 
optical yield of the AOPDF is commonly $50 \%$ on the whole spectral range. $75 \%$ can be achieved still leading to pulses in the $30 \mathrm{fs}$ range but with less shaping capacity (all results below have been obtained with $50 \%$ optical yield). However, at these high throughputs, saturation of the acoustic wave should be carefully controlled.
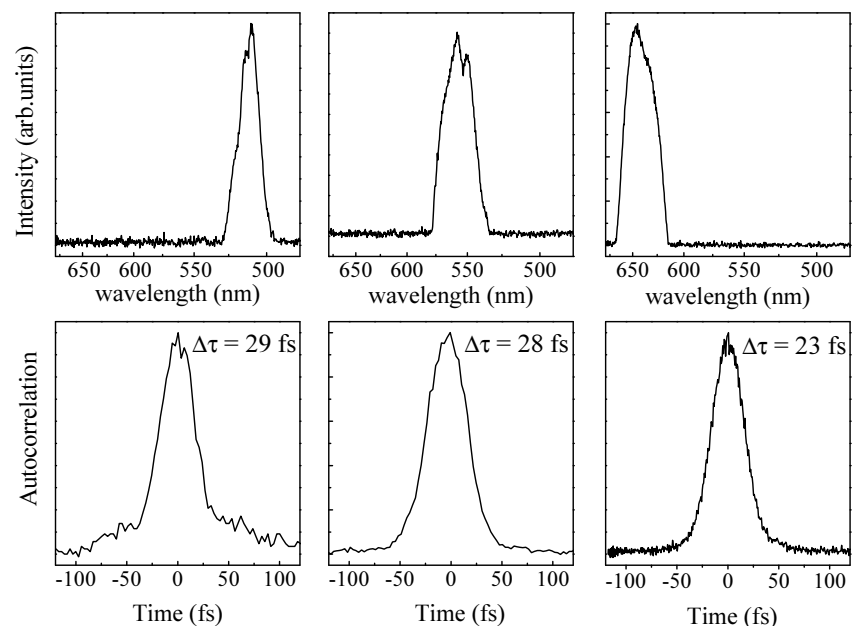

Fig. 3 Spectrum and intensity autocorrelation for various wavelengths, showing sub-30 fs pulses. Spectra have been displayed in reciprocal scale to allow direct comparison of the FWHM between the various center wavelengths.

The AOPDF is not only able to compress the pulse over the whole spectral range but also to shape it in amplitude and phase. Fig. 4 shows several cross-correlations performed in a $20 \mu \mathrm{m}$ thick BBO crystal between the output of the NOPA compressed by prisms and the output of the AOPDF. The cross-correlation signal as a function of the delay in the AOPDF is shown in Fig. 4a. The zero delay corresponds to a pulse diffracted in the middle of the crystal and positive delays to pulses diffracted on the input side of the AOPDF. From -0.5 ps to $2.5 \mathrm{ps}$ the amplitude of the signal is constant demonstrating the capacity of the AOPDF to generate delays up to 3 ps without attenuation and even 4 ps with a moderate attenuation in accordance with the computed values of Fig. 1b. For delays between -0.5 and $-1.5 \mathrm{ps}$, the cross-correlation intensity decreases as a result of acoustic absorption: the optical beam is diffracted by an acoustic pulse that propagates along a longer path in the crystal. Multiple output pulses have been generated, simply summing multiple acoustic pulses, on the whole spectral range. An example of a 5-pulse sequence is shown Fig. 4b at $640 \mathrm{~nm}$. Quadratic phase up to $4.10^{4}$ $\mathrm{fs}^{2}$ or cubic phase up to $3.10^{5} \mathrm{fs}^{3}$ have been also programmed successfully.

In this paper, the design of an AOPDF crystal optimized for shaping in the visible and the results of pulse compression and shaping experiments are presented. Optical yield up to $50 \%$ have been obtained with input energies of $6 \mu \mathrm{J}$ and an energy density of $300 \mu \mathrm{J} / \mathrm{cm}^{2}$
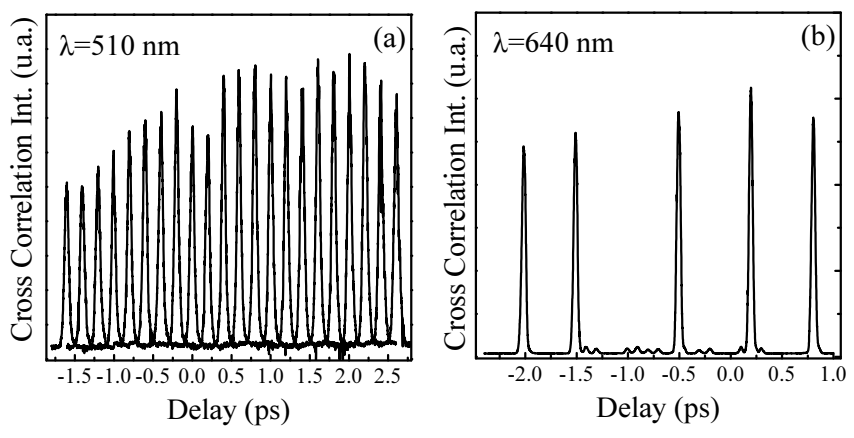

Fig. 4 Cross-correlation signal between the NOPA output compressed by prisms and the output of the AOPDF. (a) Several delays obtained every $200 \mathrm{fs}$ at $\lambda=510 \mathrm{~nm}$. (b) Multiple pulses at $\lambda=640 \mathrm{~nm}$.

without any major self phase modulation phase effect, on a never reached (for this spectral range) temporal window of 4 ps. Therefore, the AOPDF appears to be perfectly appropriate for tailoring the output of a NOPA with typical specifications of $15 \mu \mathrm{J}$ energy and $2.5 \mathrm{~mm}$ diameter FWHM. Shorter durations or higher energies can be obtained by stretching the pulse before feeding the AOPDF and using an external compressor [26]. To remain below the $\mathrm{Te}_{2}$ crystal damage threshold, an option would be to magnify the beam diameter provided that the acoustic beam inside the crystal has been scaled up. The performances in terms of maximum energy, optimal compression and temporal shaping window make it an ideal tool for tunable wavelength pulse shaping in the visible. Its large tunability $(500-650 \mathrm{~nm})$ and broad temporal window, together with its high update rate, makes this new device a unique tool for a feedback loop in optimal control experiments [1,2,3,4,10,11,27]. The authors thank Pierre Tournois for fruitful discussions.

\section{References}

1. A. Assion, T. Baumert, M. Bergt, T. Brixner, B. Kiefer, V. Seyfried, M. Strehle, and G. Gerber, Science 282, 919 (1998).

2. H. Rabitz, R. De Vivie-Riedle, M. Motzkus, and K. L. Kompa, Science 288, 824 (2000).

3. R. J. Levis, G. M. Menkir, and H. Rabitz, Science 292, 709 (2001).

4. J. L. Herek, W. Wohlleben, R. J. Cogdell, D. Zeidler, and M. Motzkus, Nature 417, 533 (2002).

5. T. C. Weinacht and P. H. Bucksbaum, Nature 397, 233 (1999).

6. J. Degert, W. Wohlleben, B. Chatel, M. Motzkus, and B. Girard, Phys. Rev. Let. 89, 203,003 (2002).

7. A. M. Weiner, Rev. Sci. Inst. 71, 1929 (2000).

8. G. Stobrawa, M. Hacker, T. Feurer, D. Zeidler, M. Motzkus, and F. Reichel, Appl. Phys. B 72, 627-30 (2001).

9. A. Monmayrant and B. Chatel, Rev. Sci. Inst. 75, 2668 (2004).

10. D. Goswami, Phys. Rep. 374, 385-481 (2003). 
11. M. Dantus and V. V. Lozovoy, Chem. Rev. 104, 18131859 (2004).

12. M. M. Wefers and K. A. Nelson, J. Opt. Soc. Am. B 12, 1343 (1995).

13. D. Zeidler, T. Witte, D. Proch, and M. Motzkus, Appl. Phys. B 74, S51 (2002).

14. H. S. Tan, W. S. Warren, and E. Schreiber, Opt. Lett. 26, 1812 (2001).

15. D. H. Reitze, A. M. Weiner, and D. E. Leaird, Appl. Phys. Lett. 61(11), 1260-2 (1992).

16. L. Xu, N. Nakagawa, R. Morita, H. Shigekawa, and M. Yamashita, IEEE J of Quant. Electr. 36(8), 893 (2000).

17. D. Zeidler, T. Hornung, D. Proch, and M. Motzkus, Appl. Phys. B 70(7), S125-31 (2000).

18. F. Verluise, V. Laude, Z. Cheng, C. Spielmann, and P. Tournois, Opt. Lett. 25, 575 (2000).

19. M. Pittman, S. Ferré, J. Rousseau, L. Notebaert, J. Chambaret, and G. Chériaux, Appl. Phys. B 74, 529 (2004).

20. A. Monmayrant, M. Joffre, T. Oksenhendler, R. Herzog, D. Kaplan, and P. Tournois, Opt. Lett. 28, 278 (2003).

21. D. Kaplan, P. Tournois, B. Chatel, and A. Monmayrant, in Ultrafast Phenomena XIV, T. Kobayashi, ed. (Springer, Niigata, 2004). To be published.

22. D. Kaplan and P. Tournois, J. Phys IV France 12, Pr569 (2002).

23. T. O. Woodruff and H. Ehrenreich, Phys. Rev. 123, 1553 (1961).

24. B. Chatel, J. Degert, S. Stock, and B. Girard, Phys. Rev. A 68, 041,402R (2003).

25. E. Riedle, M. Beutter, S. Lochbrunner, J. Piel, S. Schenkl, S. Sprlein, and W. Zinth, Appl. Phys. B 71, 457 (2000).

26. J. Seres, A. Mller, E. Seres, K. O'Keeffe, M. Lenner, R. F. Herzog, D. Kaplan, C. Spielmann, and F. Krausz, Optics Letters 28(19), 1832-1834 (2003).

27. W. S. Warren, H. Rabitz, and M. Dahleh, Science 259, $1581(1993)$ 